
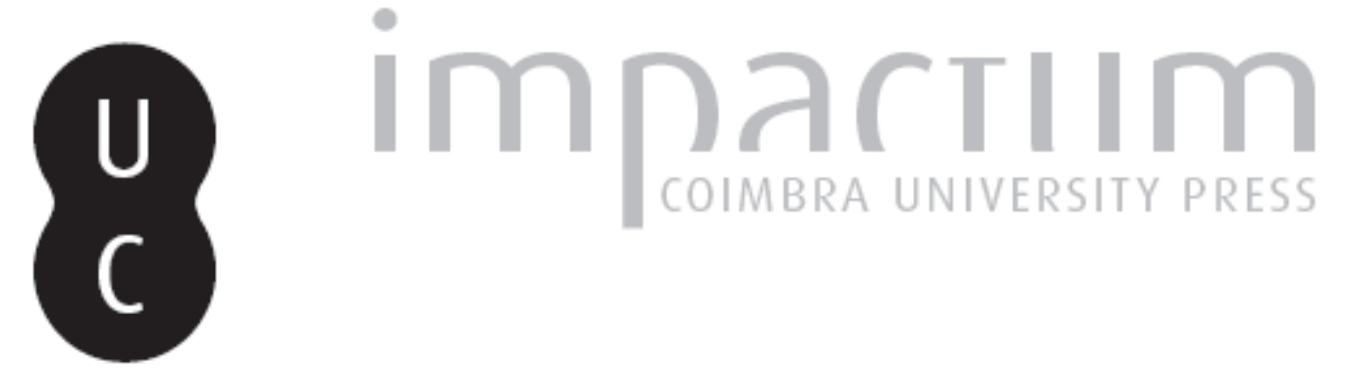

\title{
Le paradigme de la crase dans la pensée de Plutarque
}

Autor(es): Boulogne, Jacques

Publicado por: International Plutarch Society

URL persistente:

URI:http://hdl.handle.net/10316.2/37622

DOI:

DOI:http://dx.doi.org/10.14195/0258-655X_4_1

Accessed : $\quad$ 26-Apr-2023 05:39:56

A navegação consulta e descarregamento dos títulos inseridos nas Bibliotecas Digitais UC Digitalis, UC Pombalina e UC Impactum, pressupõem a aceitação plena e sem reservas dos Termos e Condições de Uso destas Bibliotecas Digitais, disponíveis em https://digitalis.uc.pt/pt-pt/termos.

Conforme exposto nos referidos Termos e Condições de Uso, o descarregamento de títulos de acesso restrito requer uma licença válida de autorização devendo o utilizador aceder ao(s) documento(s) a partir de um endereço de IP da instituição detentora da supramencionada licença.

Ao utilizador é apenas permitido o descarregamento para uso pessoal, pelo que o emprego do(s) título(s) descarregado(s) para outro fim, designadamente comercial, carece de autorização do respetivo autor ou editor da obra.

Na medida em que todas as obras da UC Digitalis se encontram protegidas pelo Código do Direito de Autor e Direitos Conexos e demais legislação aplicável, toda a cópia, parcial ou total, deste documento, nos casos em que é legalmente admitida, deverá conter ou fazer-se acompanhar por este aviso.

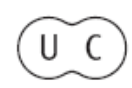


PLOUTARCHOS, n.S. Scholarly Journal of 18

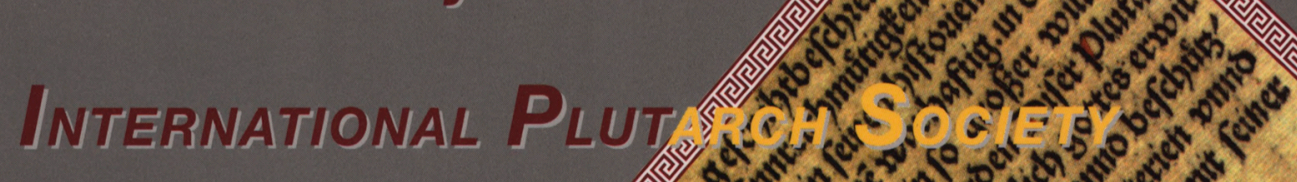

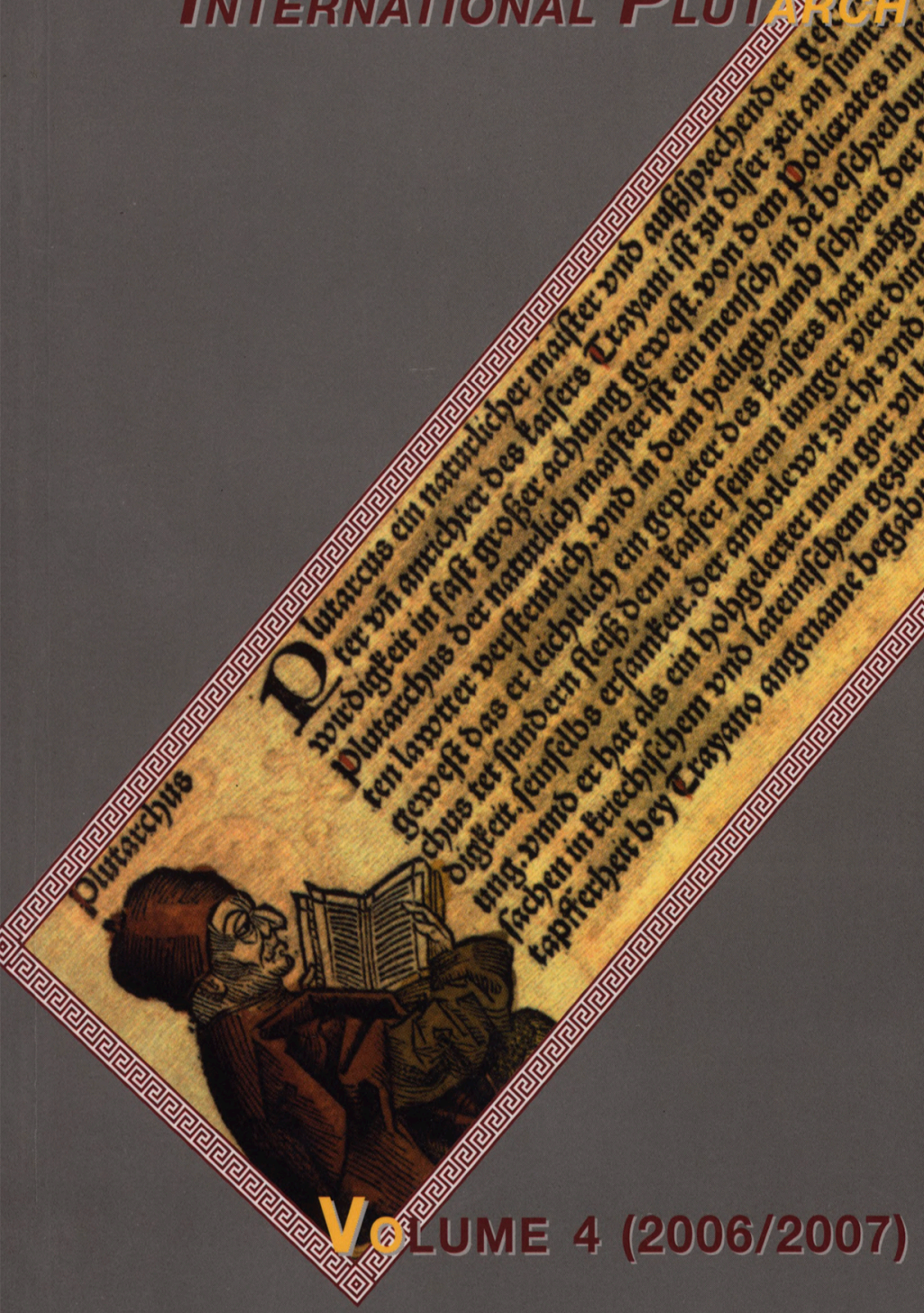

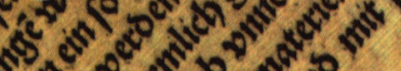

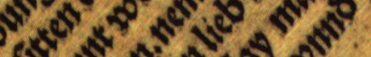

$120^{2} 0^{2} x^{2} x^{2}$

$4 \sin ^{\circ} \cos ^{\circ}$

singes

$0^{2}+0^{2} \mathrm{~s}^{\circ}$

$a^{5} x^{2}$

$s^{5}+\omega^{\circ}$

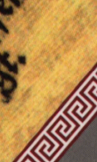

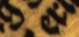

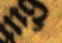




\title{
Le paradigme de la crase dans la pensée de Plutarque par \\ Jacques Boulogne \\ Université Charles de Gaulle-Lille 3 \\ HALMA-UMR 8142 CNRS
}

\begin{abstract}
Krasis as a Paradigm in Plutarch $s$ Thought

This paper aims to show that Plutarch uses the concept of krasis as a model working efficiently in various areas, such as physics, metaphysics, ethics, politics and aesthetics. In order to think perfect cohesion, he chooses the image of an integral mixing without destruction of its components parts rather that of weaving, although more current, which looks like to a change of paradigm.
\end{abstract}

Cette communication vise, dans une certaine mesure, à apporter une pièce, à mes yeux, majeure au dossier que j'ai ouvert en septembre 2000, à Montpellier, en me proposant d'étudier les structures de ce que j'ai appelé l'imaginaire métaphysique de Plutarque ${ }^{1}$ : j'entendais par là la représentation mythique des principes qui, pour lui, organisent le monde. Une conférence prononcée en mars 2002, à Liège ${ }^{12}$, m'a conduit à compléter le dossier par l'analyse de deux images organisatrices de la pensée de Plutarque, deux images non pas d'objets, mais de processus et qui, en tant que telles, jouent le rôle de paradigmes, au sens platonicien du terme ${ }^{3}$, c'est-à-dire de modèles cognitifs, qui par la comparaison rendent intelligible ce qu'on cherche à comprendre. L'une des ces deux images est celle en optique de la réflexion, à laquelle se rattache le phénomène de l'arc-en-ciel ; l'autre est celle de la crase, beaucoup plus prégnante dans l'œuvre de Plutarque. C'est sur cette dernière que je voudrais m'appesantir à présent.

Plutarque n'est pas le premier à utiliser la notion de crase. Elle se rencontre déjà chez les Présocratiques, comme le montre Elio Montanari ${ }^{4}$, mais aussi chez

1 Ma communication s'intitulait « À propos de Y Adversus Colotem. Structures de l'imaginaire métaphysique de Plutarque ».

L'imaginaire de Plutarque, publiée par la Faculté de Philosophie et Lettres de l'Université de Liège, dans la Collection « Entretiens sur l'Antiquité gréco-romaine », en 2002.

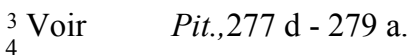

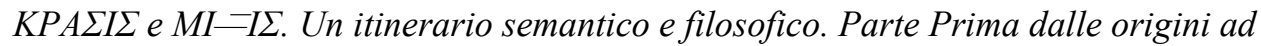
Eraclito, Quaderni dell'Istituto di Filologia Classica « Giorgio Pasquali » dell'Università degli Studi di Firenze, 1979.

PLOUTARCHOS f.s. $_{y} 4$ (2006/2007) 3-18.

ISSN 0258-655X 
les médecins, dès l'époque classique, surtout à propos des qualités élémentaires de la matière et des humeurs ${ }^{5}$. Platon en fait également usage, notamment dans le Timée, au sujet de la création de l'âme du monde, pour dire qu'elle se caractérise par un mélange complexe et intime, d'abord de la substance divisible et de la substance indivisible, puis de cette substance mixte et des deux autres $^{6}$. Mais, si sa dette envers la tradition et sans doute plus particulièrement envers Platon se révèle être indiscutable, Plutarque est, semble-t-il, le premier à se référer massivement à ce type de mélange, comme si celui-ci constituait la base de son système de représentation. De fait, on le trouve, telle une constante, aussi bien dans les domaines de la physique et de la métaphysique que dans ceux de l'éthique, de la politique et de l'esthétique, cinq domaines difficiles à distinguer tant ils s'entremêlent souvent étroitement.

Je vais donc passer en revue ces diverses références, en m'en tenant aux exemples les plus significatifs 7 . Mais, auparavant, il importe de préciser la signification que Plutarque attribue au terme de $\kappa \rho \alpha ́-$ ors et aux mots de la même famille.

\section{Un mélange homogénéisé}

Quatre passages des Euvres Morales donnent la possibilité de se faire une idée exacte de ce que Plutarque entend par крáбis.

$\mathrm{Au}$ chapitre trente-quatre des Préceptes de mariage, nous avons affaire à une typologie des mélanges. Plutarque y distingue les compositions par juxtaposition, où les éléments réunis demeurent disjoints, des assemblages, dont les composants tiennent ensemble par un ajustement mutuel, et des fusions, où l'unification des ingrédients s'effectue par soudure en coalescence.

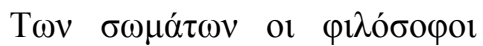

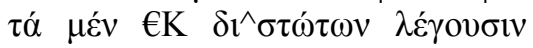

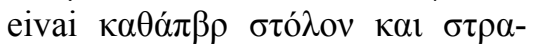

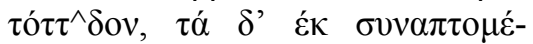

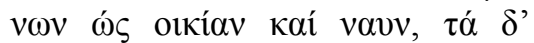

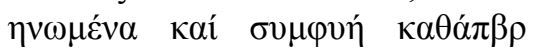

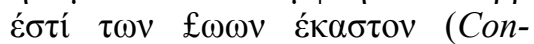
jug. praec. 142 E). Parmi les corps, les uns, aux dires des philosophes, se composent de pièces séparées tout comme une flotte ou un camp militaire, les autres de pièces attachées ensemble à la manière d'une maison ou d'un navire, tandis que d'autres encore se trouvent unifiés par coalescence tout comme

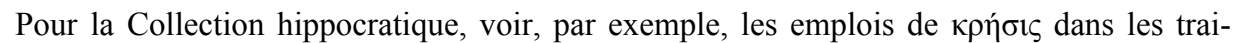
tés L'ancienne médecine (KM, 5. 4 ; 16. 1; 19. 3) et Airs, Eaux, Lieux (AER, 12. 3).

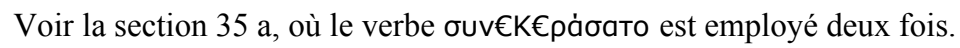

Les limites d'un article excluent l'exhaustivité, y compris pour les parties dans lesquelles se subdivisent les cinq domaines susmentionnés. Le $T L G$ relève 23 occurrences rien

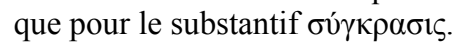


Test chacun des êtres vivants ${ }^{8}$.

Or c'est à cette troisième espèce de mélange, caractéristique de la matière vivante, que Plutarque réserve la notion de crase :

...

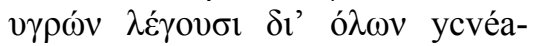

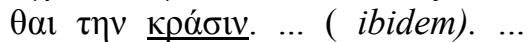
de même que les spécialistes de la nature disent qu 'il se produit une crase intégrale des liquides, ...

La crase apparaît donc comme un mélange intégral $\left(\delta \mathrm{r}^{\prime} \delta \lambda \omega v\right)$, à l'image des liquides miscibles, tels l'eau et le vin, et dont le résultat se traduit par l'apparition d'un corps composite, mais homéomère, c'est-à-dire semblable à lui-même en chacun de ses lieux.

Toutefois la fusion ne signifie pas confusion. Là dessus Plutarque partage totalement l'avis d'Aristote ${ }^{9}$ et des Stoïciens. Chacun des éléments constitu- tifs du mélange conserve en effet son intégrité et peut être retrouvé intact par décomposition; du vin se sépare de l'eau au moyen d'une éponge ${ }^{10}$, de même que l'évaporation disjoint le sel de l'eau. Si tel n'était pas le cas, nous n'aurions plus affaire à un mélange, mais à une synthèse, qui se traduit par l'altération des substances mélangées et l'apparition d'une réalité entièrement nouvelle ${ }^{11}$. Cependant, comme le signale sa critique de la théorie de Chrysippe, pour qui une seule goutte de vin se mélange à l'ensemble de la mer ${ }^{12}$, Plutarque refuse la position stoïcienne d'une compénétration. Du plein, ne saurait, à son avis, traverser du plein et devenir un enveloppé enveloppant, sans que soit présupposée l'existence de vide dans les corps, ce qui, pour lui, demeure impensable. Pourquoi, dès lors, emploie-t-il l'expression de $\kappa \rho \alpha ́ \sigma i \varsigma \delta t^{\prime}$ ó $\lambda \omega v$ qui appartient bel et bien à la terminologie stoïcienne ${ }^{13}$ ?

Les traductions sont de moi. Les textes, à quelques menues corrections près, sont tirés de la Collection des Universités de France, ou, à défaut, de la Loeb Classical Library.

Pour une présentation de la théorie aristotélicienne des mélanges et une critique des autres théories, notamment stoïciennes, voir le traité d'Alexandre d'Aphrodise De mixtione et le commentaire qu'en a publié Robert B. TodD (Alexander of Aphrodisias. On Stoic Physics. A study of the De mixtione, with preliminary Essays, Text, Translation and Commentary, Leiden, 1976).

10 Voir Philon, De confusione linguarum, 184.

11 Pour la classification stoïcienne des mélanges, voir Chrysippe, fragment 471 (v. ARNIM, $S V F$ 2, p. 151-153), où la forme extrême du mélange, la confusion ( $\left.\sigma 0 ́ \gamma \chi v \sigma ı \varsigma^{*}\right)$ débou12 che sur un produit de synthèse ( $\sigma v ́ v \theta \varepsilon \sigma 1 \varsigma)$, comme c'est le cas en pharmacopée.

12 Voir Sur les notions communes. Contre les Stö̈ciens 37, 1077 E - 1078 E et les notes 547568 de Daniel Babut (Plutarque. Euvres Morales, XV/2e Partie, CUF, Paris, 2002, pp. 297-307).

13 Voir Antipater, fragment 63 (v. ARNIM, $S V F$ 3, p. 255). 
Ce qui intéresse Plutarque dans cette formule c'est qu'elle désigne un mélange parfaitement unifié par la coextension des fluides qui le composent. Mais son contenu demeure platonicien. Nous le voyons clairement avec son exégèse du Timée, où, à propos de l'amalgame de Même, d'Autre et de Mixte créé par le Démiurge, qui stabilise la quatrième substance issue de la combinaison des trois autres en les brassant selon des proportions régies à la fois par l'association des progressions géométriques de raison 2 et de raison 3 destinées à déterminer tous les intervalles concevables et par le recours aux médiétés arithmétique et harmonique dont la fonction est de combler lesdits intervalles ${ }^{14}$, le début du chapitre 25 commence par la phrase suivante :

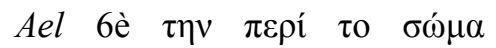

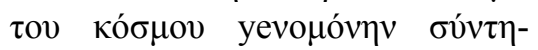

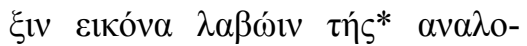

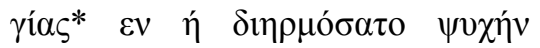
(Ani. procreat. 1025 A). Il faut prendre la fusion dont le corps est le champ comme une image de la rationalité des rapports dans lesquels le démiurge a fait contenir Tharmonisation d'une âme.
Les manuscrits de Y Epitomé (1032 E) de ce traité exégétique présentent tous le substantif $\sigma u ́ v \tau \alpha \xi ı$ (syntaxe), au lieu de

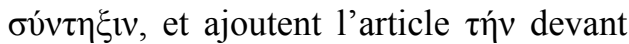

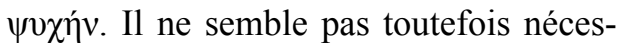
saire de corriger le texte de la version non abrégée du traité. Bien que Platon n'emploie pas le terme de $\sigma u ́ v \tau \eta \xi ı$, il évoque, en parlant de l'amitié (32 b-c) que les rapports numériques établissent entre les réalités dont ils assurent la cohésion, un mélange stable et solide ; or c'est précisément le sens, ici, du substantif $\sigma u ́ v \tau \eta \xi 1 \varsigma^{*}$, qui signifie unir étroitement en faisant fondre ensemble en un même tout. Quant à l'absence d'article, elle peut s'expliquer par la thèse que défend Plutarque dans son interprétation du mythe cosmogonique de Platon : il prête, en effet, à ce dernier le postulat qu'il existe, avant Eintervention du Démiurge, une âme précosmique, dont l'harmonisation crée le monde. Quoi qu'il en soit du texte exact ${ }^{15}$, il apparaît nettement que le mélange quasi fusionnel que constitue la crase (juste après cette phrase, Plutarque emploie le verbe $\kappa \rho \alpha \theta \eta ́ v \alpha$ pour dire qu'on ne saurait obtenir de crase pour des matières aussi

Pour l'étude de cette construction mathématique de l'âme du monde sur le modèle de la gamme musicale qu'harmonisent les rapports hémioie $(1+1 / 2)$, épitrite $(1+1 / 3)$ et épogde $(1+1 / 8)$, voir Luc BRISSON, Le même et Vautre dans la structure ontologique $d u$ Timée de Platon. Un commentaire systématique du Timée de Platon, Academia Sankt Augustin, 1994 (lue éd. Paris, 1974), p. 271-354.

15 Pour ma part, je suis le choix adopté par Harold Cherniss (Plutarch S Moralia, XIII/Part 1, The Loeb Classical Library, Cambridge (Mass.)/London, 1976) et FRANCO FERRARI et LAURA BALDI (Plutarco. La generazione delVanima nel Timeo, Introduzione, testo critico, traduzione e commento, D’Auria, Napoli, 2002). 
incompatibles que le feu et la terre sans la médiation de l'air et de l'eau, qui possèdent avec l'un et $\Gamma$ autre et entre eux des points communs, comme il en existe entre les rapports numériques ${ }^{16}$ ) ne tient pas d'une quelconque interpénétration, mais relève d'un comblement d'interstices, qui produit un effet de soudure par égalisation. Tout le dernier chapitre du commentaire développe la métaphore de rharmonie en insistant sur la notion de

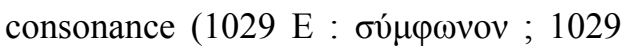

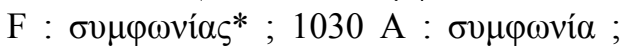
1030 B : $\sigma u \mu \varphi \omega v i ́ \alpha v)$ afin de préciser

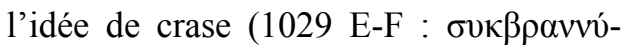
$\mu \beta v \alpha)$. Et de nouveau Plutarque puise dans le Timée $(80 \mathrm{a}-\mathrm{c})$, où Platon explique la consonance par un mouvement circulaire des sons, dans lequel les plus rapides en se ralentissant sont rattrapés par les plus lents au point que la limite finale des premiers, c'est-à-dire des aigus, finit par coïncider avec la limite initiale des seconds, en d'autres termes, des graves, ce qui procure une sensation unique, où l'aigu et le grave se perdent réciproquement l'un dans l'aut-

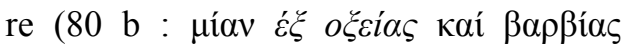

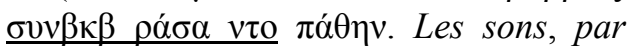
leur crase, produisent une impression unique d'aigu et de grave). Cette explication du plaisir de l'ouïe procuré par un fondu sonore, obtenu lui-même grâce à une compatibilisation (Plat, quaest.,

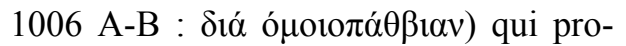

voque la crase des aigus et des graves

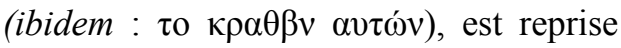
tout à la fin de la septième des Recherches platoniciennes (paragraphe 9), justement pour rendre compte de la manière dont finissent par résonner d'un même son des notes différentes.

Il ressort donc que la crase constitue pour Plutarque un mélange très particulier, propre aux fluides et qui donne lieu à une unification très intime par harmonisation, et non par suppression des différences, ce qui entraîne, grâce à la mise en solidarité ainsi créée des parties entre elles, non seulement une cohésion très stable du tout, mais encore sa parfaite homogénéité.

Ainsi conçue, la crase devient un outil conceptuel à prises multiples, qui lui permet d'unifier sa vision du monde et de l'homme.

\section{$\underline{\text { Un concept polyvalent }}$}

De fait, le modèle d'unité que figure la crase lui paraît très opératoire pour penser adéquatement le vivant, le bien et le beau.

À la fin du mythe de Sylla, dans le traité Le visage qu'on voit apparaître dans l'orbe de la lune, Plutarque explique que la vie circule de façon permanente entre les trois pôles que sont la terre, la lune et le soleil. En chacun de ces lieux se déroulent des processus

16 Voir page 1025 A-B. Sur l'application à la cohésion du corps matériel du monde de l'image de la crase utilisée pour caractériser le mélange de l'âme cosmique, voir la note 181 de F. FERRARI (op. cit. supra, p. 302-303). 
décisifs, surtout sur la terre et sur la lune. En effet, sur cette dernière se produisent aussi bien l'union de la psukhé, dont la substance est lunaire, et du nous, qui provient du soleil, que leur désunion, le nous se séparant en une seconde mort de la psukhé pour regagner sa source lumineuse, le soleil. Sur la terre, nous assistons également à ces deux processus, l'ensemble nous-psukhé se mêlant, d'un côté, à un corps et, de l'autre, se libérant de lui en une première mort. Plutarque reprend la figure mythologique des Moires et affecte chacune à l'un de ces trois pôles, afin qu'en tant que divinités du destin elles président à la bonne circulation de la vie. Or il transforme leur activité : de fileuses qu'elles sont traditionnellement, elles deviennent des brasseuses :

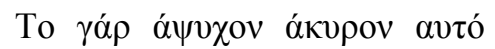

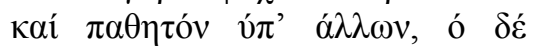

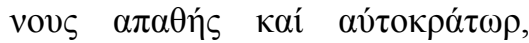

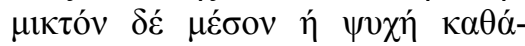

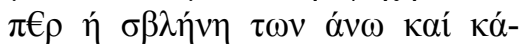

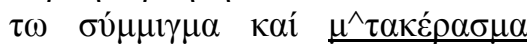
v́to

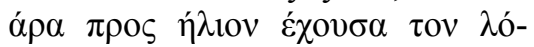

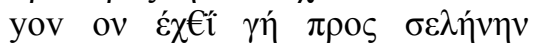
$\{$ De facie 945 C-D). Car, si Гinanimé est par lui-même incapable de domination et reste passif dans ce que lui fait subir ce qui lui est autre, Fintellect est dépourvu de passivité et ne dépend que de luimême, tandis que Vâme est une chose mixte et intermédiaire, tout comme la lune a été générée par le dieu pour assurer un mélange intime des réalités supérieures et des réalités inférieures et un transvasement qui assure leur crase, en ayant donc relativement au soleil ce rapport que la terre a relativement à la lune.

Mis à part Atropos, qui lance le mouvement, Clotho et Lakhésis réunissent, non pas en tissant ${ }^{17}$, mais en mélangeant, et l'intimité de leurs mélanges ( $\sigma \dot{\mu} \mu \imath \gamma \mu \alpha)$ appelle l'image de la crase $(\mu € \tau \alpha \kappa \varepsilon \dot{\varepsilon} \rho \sigma \mu \alpha)^{18}$.

Celle-ci fonctionne donc à la manière d'un schème d'intelligibilité, qui permet de se représenter le mystère de la parfaite unification de substances aussi hétérogènes que le corps, la psukhé et le nous, ainsi que la cohésion du monde ${ }^{19}$.

Un autre mythe, celui de Thespésios, dans Les délais de la justice divine, au sujet de l'oracle de la Nuit et de la Lune,

Comme c'est le cas dans le pseudo-Plutarque De fato ( 568 E).

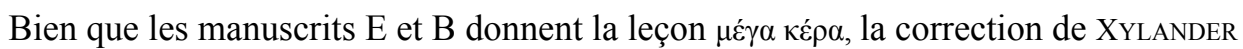
et AmYot, adoptée par WytTenBach et H. Cherniss \{Plutarch s Moralia, XII, The Loeb Classical Library, Cambridge (Mass.)/London, 1968, lert éd. 1957), semble aller de soi.

Cf. De genio Socratis (591 B), où nous retrouvons les trois Moires en des positions respectives légèrement différentes (Atropos est située dans l'invisible, Clotho dans la région du soleil, Lakhésis dans celle de la lune). Nous avons donc affaire, dans le $D e$ facie, à un décalage vers la terre du dispositif général censé maintenir la cohésion du monde ; l'ensemble de l'architectonique n'est pas remis en question. 
visité par Orphée lors de sa quête ouranienne d'Eurydice, utilise à nouveau l'image du brassage. 11 s'agit, cette fois, d'un vaste cratère où se jettent des courants de lumière de teintes différentes, mais que trois démons uniformisent en une seule couleur, la couleur blanche, par l'intermédiaire de certaines proportions.

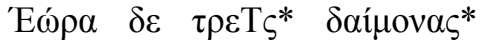

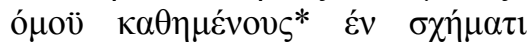

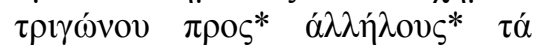

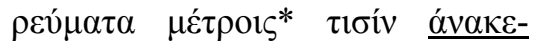

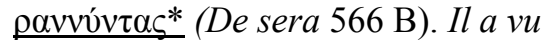
trois démons assis au même endroit, dans une disposition qui formait entre eux un triangle, en train de procéder à la crase des courants, au moyen de certaines mesures.

L'introduction, ici, de l'idée de mesure fait écho à la manière dont le Démiurge du Timée homogénéise l'âme du monde.

Autre exemple eschatologique, celui $\mathrm{du}$ dialogue Le démon de Socrate. Timarque y raconte qu'il a vu, lors du voyage de son nous dans l'Au-delà, que les âmes ne se mélangent pas toutes à la chair de la même manière ; les unes s'enfoncent intégralement dans le corps, 2021 dont elles subissent entièrement les passions ; les autres ne connaissent cette crase que partiellement.

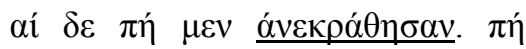

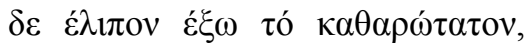

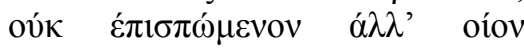

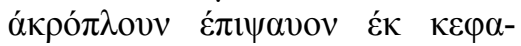

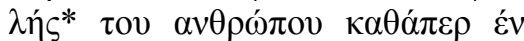

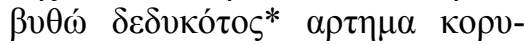

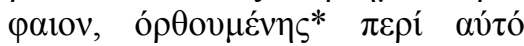

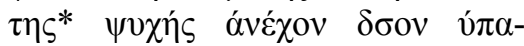

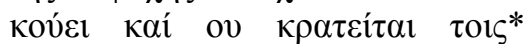
$\pi \alpha ́ \theta \varepsilon \sigma u v$ (Gen. Soc. 591 D-E). Les autres, d'un côté, s y étaient mélangées en formant une crase, mais, de l'autre, avaient laissé en dehors ce qu 'il y a de plus pur, qui, du coup, au lieu d'être entrầné, à l'instar d'un objet suspendu tout en haut, flottant en surface et qui affleure de la tête de l'homme alors que celui-ci se trouve enfoncé dans les profondeurs, tient droit autour de lui tout ce qui dans l'âme lui obéit et $n$ 'est pas dominé par les passions.

Le modèle utilisé par Plutarque pour imaginer l'union de l'âme et du corps se révèle donc être celui des fluides qui se mêlent en un tout homogène, et non pas celui de l'articulation de solides entre eux.

Ce sont des démons encore qui dans le phénomène des oracles sont imaginés en train de

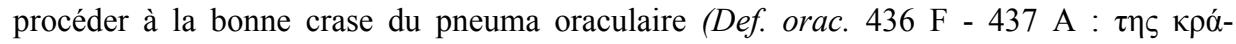
$\sigma \varepsilon \omega \varsigma \tau \alpha u ́ \tau \eta \varsigma)$.

21 Nous n'ouvrirons pas, ici, le complexe dossier de la démonologie, bien qu'on y retrouve la notion de крáбıs, comme, entre autres, dans le De defectu oraculorum (436 F - 437 A), où le phénomène de la divination est expliqué par un mélange intime sous l'effet de l'action du soleil entre l'âme humaine et le souffle exhalé par la terre, une crase à l'harmonisation de laquelle sont préposés les démons. 
En apporte la confirmation un fragment du traité Le désir et le chagrin relèvent-ils de Lâme ou du corps ? Plutarque y explique, en effet, que la comparaison avec les récipients n'est pas pertinente, car il est impossible de délimiter nettement, comme c'est le cas pour un contenu et son contenant, où s'arrête l'âme et où commence le corps auquel elle est intimement associée. La nature, dit-il, efface les lignes de démarcation et de deux substances elle n'en forme plus qu'une : le mélange est si intime qu'il constitue une crase unifiante.

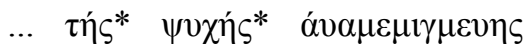

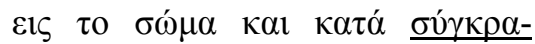

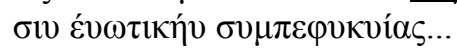

(Lihid. 6). ...comme Pâme se trouve intimement mêlée au corps et dans un état de coalescence par une crase unifiante ...

$\mathrm{Si}$ nous passons au domaine de la médecine nous retrouvons naturellement l'image, qui est entrée depuis longtemps dans la terminologie de la tradition médicale. Par exemple, au chapitre 26 des Préceptes de santé, Plutarque insiste sur le devoir pour chaque individu d'apprendre à connaître sa crase corporelle, c'est-à-dire ce qu'on appelle encore souvent le tempérament, lequel consisterait en particulier en un mélange faisant 22 fusion des qualités élémentaires de la matière, tel le chaud et le sec. Il ne faut, écrit-il, ... ni ignorer une crase, celle que présente le corps en matière de chaleur et de sécheresse ...

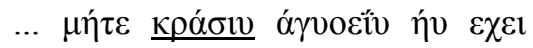

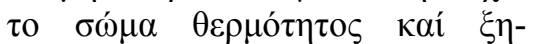

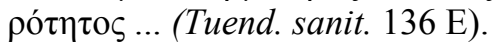

Transposée dans la sphère de l'éthique, la valeur opératoire de l'image conserve toute son efficacité. Le traité La vertu morale nous le fait constater. S'en prenant à l'idéal stoïcien de l'apathie, Plutarque, dans le prolongement de la théorie aristotélicienne de la métriopathie, définit la bonne santé morale en ces termes :

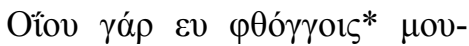

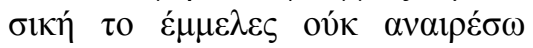

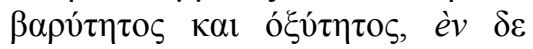

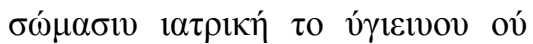

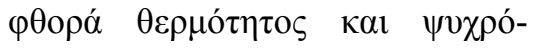

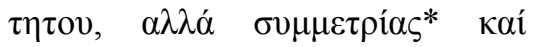

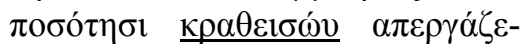

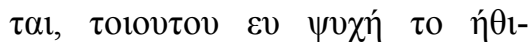

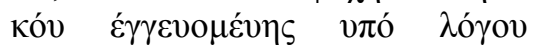

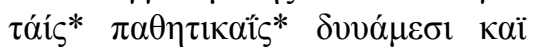

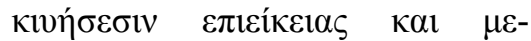

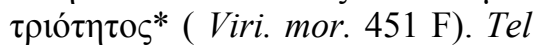
$l$ 'accord qui, dans les sons, $n$ 'est pas produit par la musique grâce à la suppression des graves et des aigus et telle la bonne santé qui,

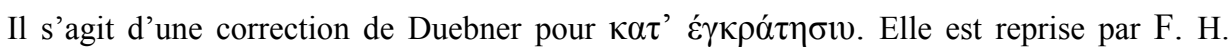
SANDBACH (Plutarch $S$ Moralia, XV, The Loeb Classical Library, Cambridge (Mass.)/London, 1969, p. 48) et ne fait aucun doute, étant donné le contexte.

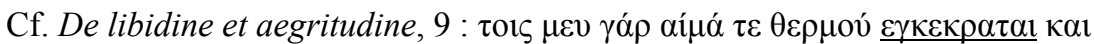

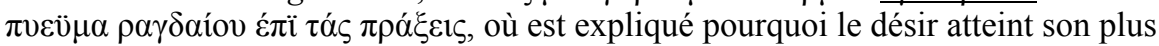
haut degré d'intensité chez les jeunes. 
dans le corps, ne l'est pas grâce à la destruction de la chaleur et du froid, mais grâce à la commensurabilité des quantités, quand il y a crase, telle est produite l'éthique dans l'âme, si la raison introduit dans la puissance des passions et dans leurs mouvements modération et mesure.

Ici, la métaphore devient normative. La crase implique des dosages calculés sur le modèle de l'harmonie musicale, qui réunit en un tout et sans discordance les notes situées aux deux extrémités de la gamme. De la même manière l'excellence du comportement doit utiliser la vertu motrice des émotions et des passions, et non pas éliminer ces dernières, à condition toutefois de les rendre cohérentes entre elles et de les concilier avec leur contraire, la pensée rationnelle, et de les subordonner à ses exigences.

Si l'on aborde la vie sociale, le paradigme de la crase fonctionne à plein pour suggérer à quelle forme d'union il convient d'aspirer, qu'il s'agisse du couple, la base de toute communauté, ou de peuples.

Le mariage constitue, aux yeux de Plutarque, une unité duale particulièrement résistante aux épreuves du devenir, s'il repose sur un mélange qui fait fusion. Il convient, en effet, explique-t-il dans ses Préceptes de mariage, de dépasser le stade de ceux qui couchent ensemble par intérêt matériel ou même par désir d'une postérité, car alors on a affaire à une simple cohabitation, sans symbiose.

$\Delta \varepsilon \imath \quad \delta \varepsilon, \quad \omega ́ \sigma \pi \varepsilon \rho$ oí $\varphi v \sigma 1 \kappa o i ́$

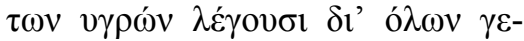

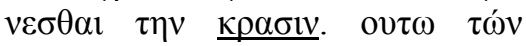

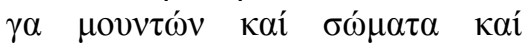

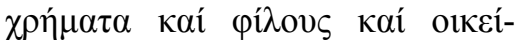

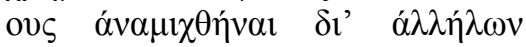
(Conjug. praec. 142 F - 143 A). De même que les spécialistes de la nature disent qu'il se produit une crase intégrale des liquides, il faut de même que corps, richesses, amis aussi bien que parents, pour ceux qui se marient, se mêlent intimement entre eux.

Le secret de cette crase réside, selon Plutarque, dans l'amour, comme il l'expose dans son Dialogue sur Eros, en prenant pour repoussoir les agrégats instables formés par les atomes de la physique épicurienne.

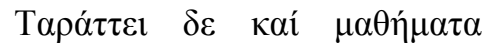

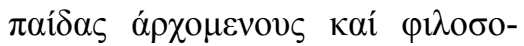

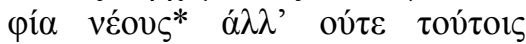

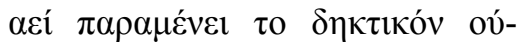

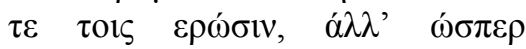

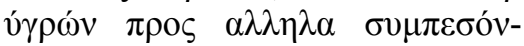

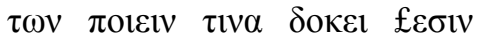

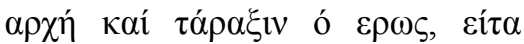

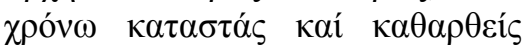

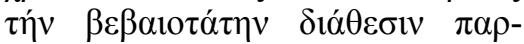

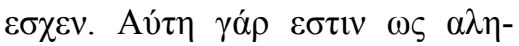
$\theta \omega ́ \varsigma \eta ́ ~ \delta \imath^{\prime}$ ó $\lambda \omega v \lambda \varepsilon \gamma \sigma \mu \varepsilon v \eta \underline{\kappa \rho \alpha-}$

À nouveau, il est difficile de ne pas penser à Platon, cette fois au discours d'Éryximaque dans le Banquet (180 d-e), où la médecine se définit comme l'art de faire régner la concorde entre les contraires. 


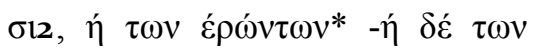

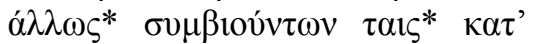

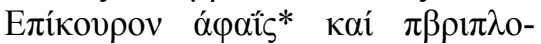

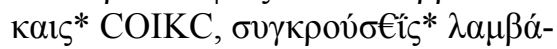

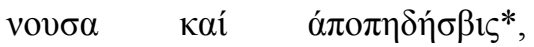

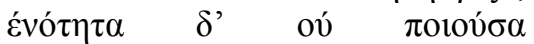

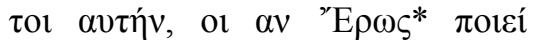

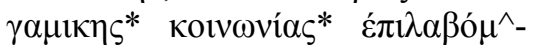
$\operatorname{vos}^{*}$ \{Amat. 769 E-F). C'est un trouble aussi que produisent les mathématiques chez les enfants qui en commencent l'étude et la philosophie chez les jeunes ; mais leurs morsures ne subsistent pas toujours, ni pour eux, ni pour ceux qui s'aiment; comme lorsque des liquides se rencontrent, l'amour semble au commencement provoquer quelque bouillonnement et quelque trouble, puis avec le temps il reprend son assiette, se clarifie et présente la disposition la plus stable. En effet on a alors affaire à ce qu 'on appelle la crase intégrale, celle de ceux qui s 'aiment, tandis que la rencontre de ceux qui vivent ensemble autrement ressemble aux contacts et aux entrelacements dont parle Epicure, avec des entrechoquements et des rebonds, sans produire une unité telle qu'en produit Eros quand il $s$ 'empare de la vie commune des époux.
Faisant de la cohésion conjugale un facteur décisif de la solidité d'une société, Plutarque pense que l'hégémonie de Rome tient pour une bonne part à la remarquable stabilité du mariage romain, dont l'origine remonte au rapt des Sabines, un acte de violence compensé par une telle multiplication de marques d'honneur, d'attention et de bienveillance que le temps a fini par créer entre elles et leurs agresseurs une union fusionnelle.

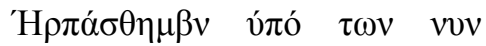

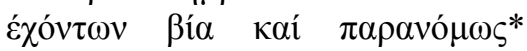

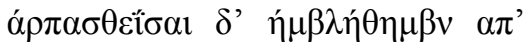

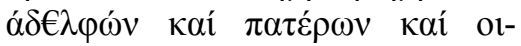

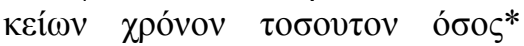
$\eta \dot{\eta} \mu \alpha \varsigma^{*} \pi \rho \circ \varsigma^{*} \tau \alpha \dot{\varepsilon} \chi \dot{\chi} \theta 1 \sigma \tau \alpha \underline{\kappa^{\wedge} \rho \alpha ́ \sigma \alpha \tau}$

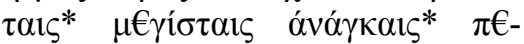

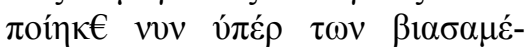

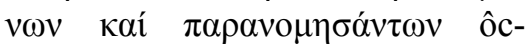

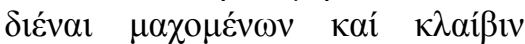
$\theta v \eta \sigma \kappa o ́ v \tau \omega v$ \{Rom. 19.4). Nous avons été enlevées de force et contrairement aux lois par ceux à qui maintenant nous appartenons, mais, après notre enlèvement, nous avons été si longtemps oubliées de frères, de pères et de parents que, formant par les contraintes les plus grandes une

Voir Quaest. rom., 267 C (cf. 278 E et la Sunkrisis Lycurgue-Numa, 3.13), où Plutarque rapporte que les Romains ont gardé le souvenir de Spurius Carvilius parce qu'il fut le premier à divorcer, 250 ans après la fondation de l'Vrbs, et encore n'était-ce qu'en raison de la stérilité de son épouse (voir Sunkrisis Thésée-Romulus, 6.4).

Sur le sens que Plutarque donne à cet épisode légendaire de l'histoire de Rome, voir JACQUES BOULOGNE, " L'utilisation de l'enlèvement des Sabines chez Plutarque ", Bulletin de EAssociation Guillaume Budé, (2000/4) 353-363. 
erase avec ce qu 'il y avait de plus hostile, nous éprouvons maintenant de la crainte, quand ils combattent, pour ceux qui nous ont fait violence au mépris des lois, et nous pleurons quand ils meurent.

L'affection, par le respect qu'elle implique, non seulement conduit au même résultat que l'amour, mais en l'occurrence elle permet aussi la erase socio-politique des Romains et des Sabins qu'espérait réaliser Romulus grâce aux mariages mixtes ${ }^{27}$ et à un mélange des familles facteur d'une fusion inter-ethnique.

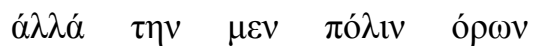

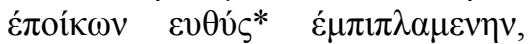

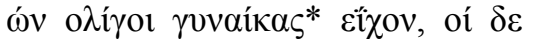

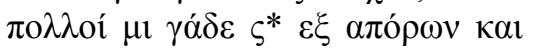

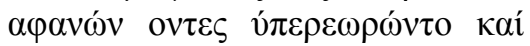

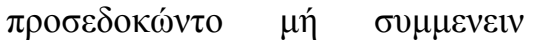

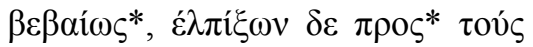

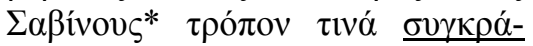

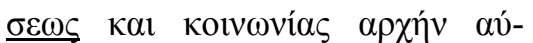

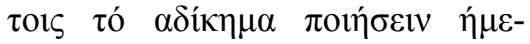

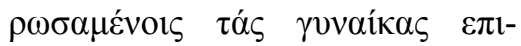

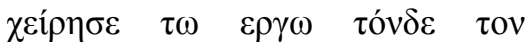

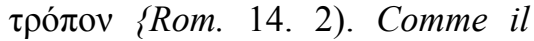
voyait sa ville se remplir aussitôt d'étrangers dont un petit nombre seulement avait des épouses, alors que la plupart étaient des ramassis d'individus sans ressources et obscurs, ce qui leur valait d'être méprisés et d'être tenus pour incapables de rester solidement ensemble, et comme il espérait que le tort créerait pour eux d'une certaine manière avec les Sabins un commencement de erase et de communauté, s'ils parvenaient à apprivoiser leurs épouses, il se mit au travail de la manière suivante.

Il n'est pas inintéressant de noter que Plutarque attribue au temps ce même rôle d'opérateur de fusion, quand il voit dans l'hégémonie romaine l'effet d'un processus historique unifiant, voulu par la Providence pour sortir la terre habitée $\mathrm{du}$ chaos politique et $\mathrm{y}$ introduire un ordre harmonieux sur le modèle platonicien de l'âme du monde.

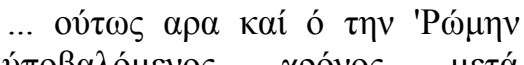

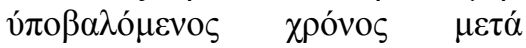

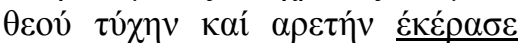

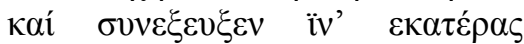

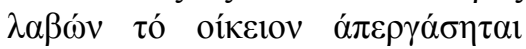

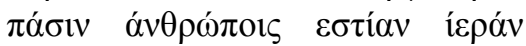

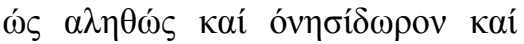

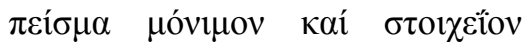

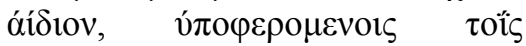
$\pi \rho \alpha ́ \gamma \mu \alpha \sigma \mathrm{iv} \quad \dot{\alpha} \gamma \kappa v \rho \eta \beta o ́ \lambda i o v \quad \sigma \alpha ́ \lambda o v$

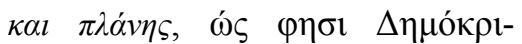
tos \{Fort. rom. 316 F-317 A). ... de même le temps qui a posé les fondements de Rome a également, avec l'aide du dieu, fait une erase de la fortune et de l'excellence et il

27 Plutarque croit beaucoup à la vertu des mariages mixtes pour Funification des civilisations, comme il le laisse également transparaître en louant Alexandre d'avoir mélangé de

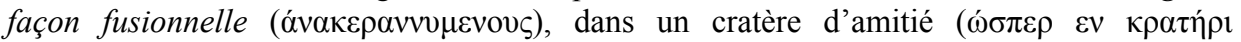

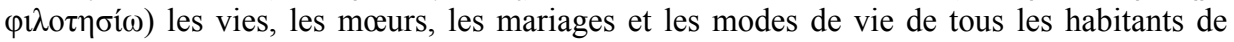
la terre (Alex. fort. 329 C-D). 
les a conjuguées afin de $s$ *emparer de ce que chacune des deux possède en propre et de réaliser pour tous les hommes un foyer véritablement sacré et bienfaiteur, une amarre fixe et un élément de base éternel, un ancrage, comme I'<affirme Démocrite ${ }^{2 *}$, contre $l$ 'agitation des flots et les errances, lors des vicissitudes des affaires humaines.

La réussite de Rome est ainsi mise sur le compte d'un alliage parfaitement homogène de chance et de mérites, lequel alliage qualifie précisément l'Empire pour l'unification harmonieuse de l' oikoumène.

Les exemples précédents nous ont déjà fait entrer dans le champ du politique, considéré par Plutarque comme $\Gamma$ activité la plus élevée en dignité . Ainsi, pour donner une idée de l'importance de la fonction d'un chef d'État, le compare-t-il ${ }^{30}$ à l'action cosmétique du soleil qui met absolument tout en beauté et mélange à tout de façon fusionnelle ce charme envoûtant qui émane de lui.

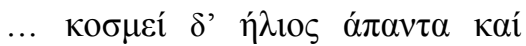

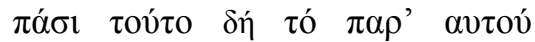

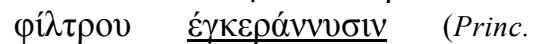
iner $780 \mathrm{E})$.... quant au soleil, il met absolument tout en beauté et de tout il procède à la erase avec ce charme envoûtant qui émane de lui.

L'embellissement apporté par la lumière solaire ne tient pas, ici, de la parure factice et superficielle ; l'ensorcellement des yeux provient de la révélation de la beauté du monde dont il provoque le rayonnement, si bien que son pouvoir de séduction se retrouve incorporé dans toutes les réalités éclairées de ses rayons. Loin d'être accessoire, la recherche du beau doit être regardée comme la fin de l'action politique. Pour y parvenir, le chef d'Etat a l'obligation de bien gouverner, en évitant notamment les deux fautes que constituent l'excès de complaisance et l'excès de dureté. Il lui faut allier douceur et fermeté, autrement dit il doit réunir en une erase personnelle les qualités illustrées aussi bien par un Phocion que par un Caton le Jeune, à savoir l'humanité et la sévérité, mais également la sécurité et la bravoure, le souci d'autrui et pour son propre compte l'intrépidité, les précautions contre le mal et la recherche énergique de la justice, toutes vertus dont le mélange par fusionnement ( $\gamma^{\prime} \gamma \varepsilon \kappa \rho \alpha \mu \varepsilon \dot{-}$ vov) produit un même et unique caractère éthique ${ }^{32}$. Cette conception du bon

28 Fragment B 148 DK. Cf. Plutarque, De amore prolis 495 E.

29 Voir le traité De liberis educandis (7 F - 8 B) ; cf. Lat. viv. 1129 B.

30 Voir Adprincipem ineruditum $780 \mathrm{D}-\mathrm{F}$.

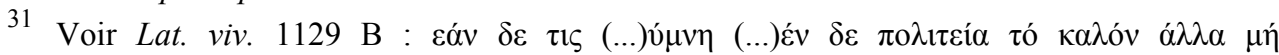

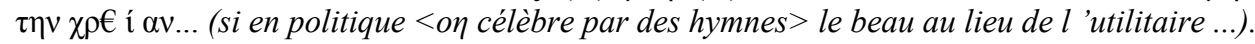

Voir Phoc. 3. 8. 
gouvernement est décrite au moyen de l'image cosmique de la course du soleil.

' $\Omega \sigma \pi \varepsilon \rho$ ovv $\tau$ ov

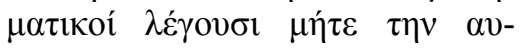

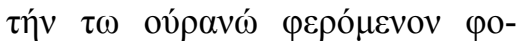

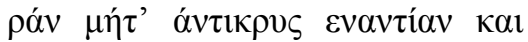

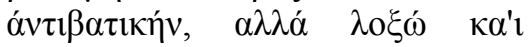

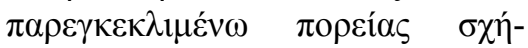

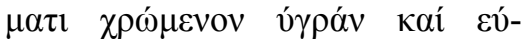

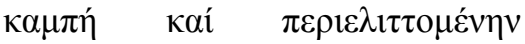

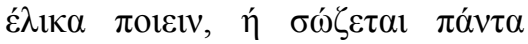

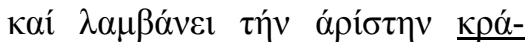

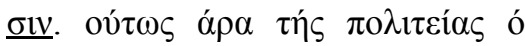

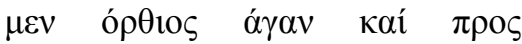

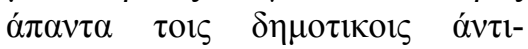

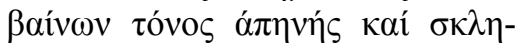

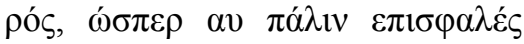

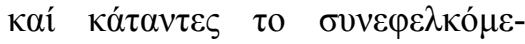

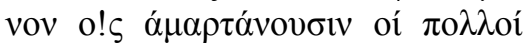

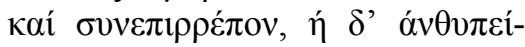

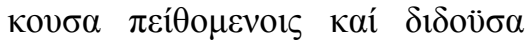

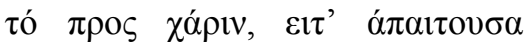

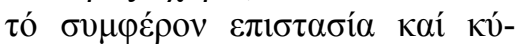
$\beta \varepsilon \quad \rho v \eta \sigma 1 \zeta \quad(\alpha ́ v \theta \rho \omega ́ \pi \omega v \quad \pi 0 \lambda \lambda \dot{\alpha}$

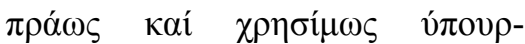

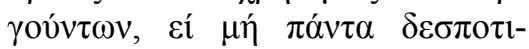

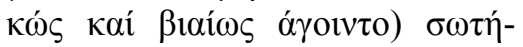

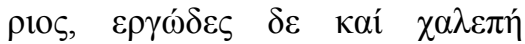

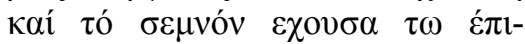

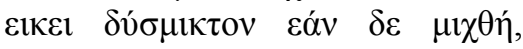

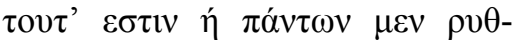

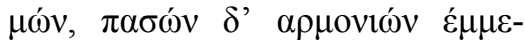

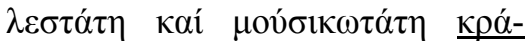

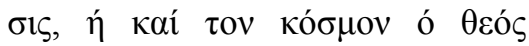

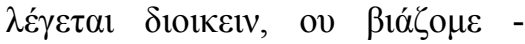

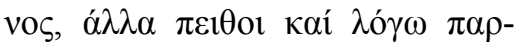

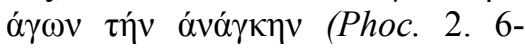
9). De même donc que le soleil, aux dires des mathématiciens, ne se déplace $n i$ du même déplacement que le ciel, ni d'un déplace- ment directement opposé et contraire, mais en utilisant une figure de cheminement oblique et inclinée pour décrire une hélice souple et aux belles courbes, qui assure la conservation de tout et procure à tout la meilleure crase, de même en politique la tension trop droite et trop contraire aux passions populaires est raide et dure, comme inversement se laisser entraîner par les erreurs de la foule et suivre les inclinations de celle-ci c'est se retrouver sur une pente glissante, alors que la direction et le gouvernement qui cèdent un peu à ceux qu'on cherche à persuader et donnent ce qui leur fait plaisir, pour ensuite réclamer d'eux ce qui est utile (car les hommes rendent souvent service avec gentillesse et de façon utile, dès lors qu'ils ne sont pas en tout conduits despotiquement et avec violence), sont salutaires, mais exigeants, pénibles et dotés d'une solennité qui se mêle difficilement à l'indulgence; or, si elle parvient à s'y mêler, on a alors la crase la mieux accordée et la plus musicale de tous les rythmes et de toutes les harmonies, celle précisément au moyen de laquelle le dieu, dit-on, administre le monde sans violence, mais en y glissant la nécessité par le biais de la persuasion et de la raison.

Assimilée à l'action divine, l'action politique idéale harmonise donc le monde des hommes, en effectuant une crase conçue sur le modèle de l'harmonie musicale, qui agit obliquement sur le 
comportement humain pour introduire dans les sociétés l'indispensable solidarité et pourvoir de la sorte à leur cohésion en réussissant à produire la concorde, grâce à la combinaison des contraires $^{33}$. C'est ce que symbolise également l'allusion à ce déplacement hélicoïdal du soleil sur le plan incliné de l'écliptique, une marche qui conjugue avec la souplesse du vivant le mouvement circulaire et le mouvement rectiligne.

$\mathrm{Si}$ je me suis autorisé une citation aussi longue avec cette page de la préface de la Vie de Phocion, c'est parce qu'elle récapitule magnifiquement les trois dimensions principales de l'imaginaire plutarquien, théologique, politique et esthétique, avec son évocation de l'unité organique d'un être vivant harmonieux, épargné par l'entropie de la corruption. Pour Plutarque, le critère du beau est fourni par l'alliage des contraires, qui transforme la matière inerte en matière vivante, un mélange tel qu'il soude entre elles ses composantes sans qu'apparaisse la moindre trace de soudure ${ }^{34}$.

Il est, à ce sujet, significatif que nous rencontrions encore la notion de erase à propos de la perfection du style. Pour justifier sa préférence pour Ménandre aux dépens d'Aristophane, Plutarque écrit que son phrasé réussit par sa erase à homogénéiser en une unité sans faille l'expression d'une grande variété de caractères.

H 8è M $\beta v \alpha ́ v \delta \rho o v ~ \varphi \rho \alpha ́ \sigma ı \varsigma^{*}$ ov-

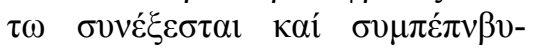

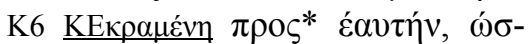

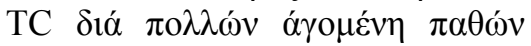

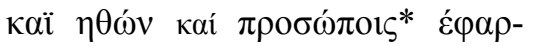

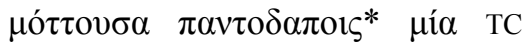

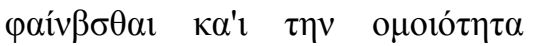

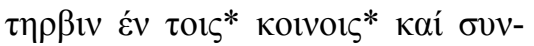

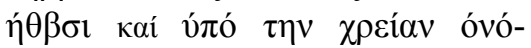

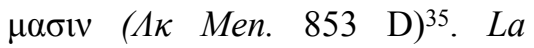
phrase de Ménandre est si polie et d'une erase qui la met tellement à Vunisson avec elle-même que, tout en traversant des passions et des caractères en grand nombre et tout en restant en harmonie avec des personnages de toute sorte, elle apparaît une et conserve sa similitude dans le vocabulaire commun, ordinaire et usité.

On peut même se demander si Plutarque lui-même ne procède pas à un mélange par fusionnement de ce genre, quand il combine, par la multiplication des citations, en une seule et même voix, sa propre parole et celle des philosophes, des poètes et des politiques qu'il a l'habitude

Cf. Galb. 1.3.

Voir J. Boulogne, « La poétique de Plutarque : une esthétique du fondu », dans

Studies in Ancient Literary Theory and Criticism. Essays edited by Jerzy STYKA, Classica Cracoviensia V, Krakow, 2000, 169-179.

35 Guy Lachenaud (Plutarque. CEuvres Morales. Traités 54-57, CUF, Les Belles Lettres, Paris, 1981, p. 93) range sans hésiter ce fragment au nombre des ouvrages authentiques de Plutarque. 
de mêler intimement dans ses ouvrages ${ }^{36}$.

$\mathrm{Au}$ terme de cette investigation, dont je rappelle qu'elle n'a rien d'exhaustif, je conclurai très brièvement sur les deux remarques programmatiques suivantes. Plutarque, d'abord, semble abandonner la métaphore du tissage, pourtant massivement présente dans la tradition de la pensée antique ${ }^{37}$, au profit de celle de la erase. Ensuite, ce changement de paradigme chez lui peut découler d'une influence de la pensée gnostique, telle qu'elle s'exprime dans les Livres dits d'Hermès, dont il nous apprend, dans sa somme d'herméneutique religieuse, Is is et Osiris (375 F), qu'il les a lus. C'est de cette lecture qu'il faudrait étudier les traces dans l'ensemble de son œuvre.

\section{BIBLIOGRAPHIE}

\section{BABUT, D.,}

Plutarque. Euvres Morales, XV/T Partie. Sur les notions communes, Contre les Stoïciens, CUF, Les Belles Lettres, Paris, 2002.

Boulogne, J.,

- «L'utilisation de l'enlèvements des Sabines chez Plutarque », Bulletin de l! Association Guillaume Budé, (2000/4) 353-363.

- «La poétique de Plutarque : une esthétique du fondu », in J. STYKA (éd.), Studies in Ancient Literary Theory and Criticism, Classica Cracoviensia V, Krakow, 2000, pp. 169-179.

BRISSON, L.,

Le même et l 'autre dans la structure on- tologique du Timée de Platon. Un commentaire systématique du Timée de Platon, Academia Sankt Augustin, 1994 (lère éd. Paris, 1974).

Cherniss, H.,

- Plutarch s Moralia, XHI/Part 1, The Loeb Classical Library, Cambridge (Mass./London, 1976.

- Plutarch s Moralia, XII, The Loeb Classical Library, Cambridge (Mass.)/ London, 1968 (lere éd. 1957).

Ferrari, F. \& Baldi, L.,

- Plutarco. La generazione delPanima nel Timeo, Introduzione, testo critico, traduzione e commento, D'Auria, Napoli, 2002.

Lachenaud, G.,

- Plutarque. Euvres Morales, XII/1. Traités 54-57, CUF, Les Belles Lettres, Paris, 1981.

Montanari, E.,

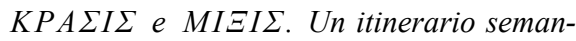
tico e filosofico. Parte Prima dalle origini ad Eraclito, Quademi dell'Istituto du Filologia Classica «Giorgio Pasquali » dell'Universita degli Studi di Firenze, Firenze, 1979.

SANDbach, F. H.,

- Plutarchs Moralia, XV, The loeb Classical Library, Cambridge (Mass.)/London, 1969.

SCHeId, J. \& SVenbro, J.,

Le métier de Zeus. Mythe du tissage et du tissu dans le monde gréco-romain, Paris, 1994.

Topd, R. B.,

Alexander of Aphrodisias. On Stoic Physics. A study of the De mixtione, with preliminary Essays, Text, Translation and Commentary, Leiden, 1976.

Voir deux études de J. BOULOGNE à paraître, l'une aux Éditions Ousia de Bruxelles sur le jeu des citations dans $L E$ de Delphes, et l'autre sur l'éclectisme du traité Préceptes de santé dans les Actes du Congrès de l'APLAES qui s'est tenu à Lille en mai 2003.

Voir l'essai de JOHN SCHEID et JeSPER SvENBRO, Éd. La Découverte, Paris, 1994. 